
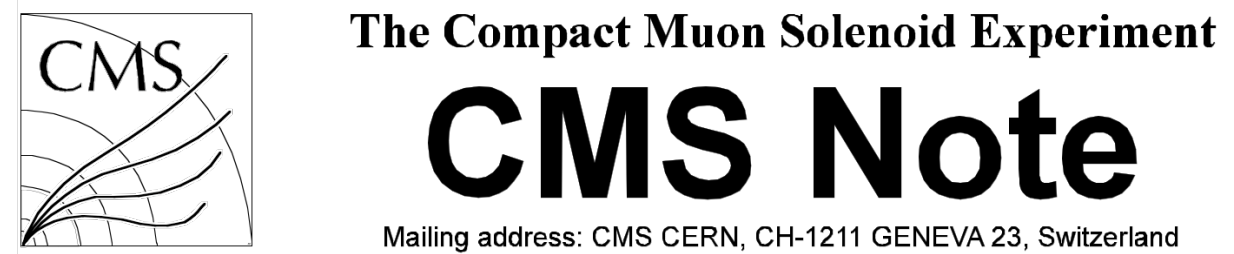

Mailing address: CMS CERN, CH-1211 GENEVA 23, Switzerland

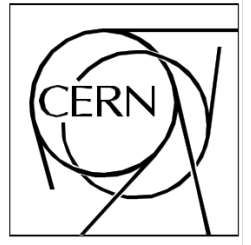

November 20, 2006

\title{
Energy Resolution of the Barrel of the CMS Electromagnetic Calorimeter
}

P. Adzic ${ }^{c}$, R. Alemany-Fernandez ${ }^{m}$, C. B. Almeida ${ }^{m, n}, N$. M. Almeida ${ }^{m}, G$. Anagnostou ${ }^{b}$, M. G. Anfreville ${ }^{k}, I$. Anicin ${ }^{c}, Z$. Antunovic ${ }^{\text {ad }}$, E. Auffray ${ }^{j}, S$. Baccaro ${ }^{a b}, a^{a}, S$. Baffioni ${ }^{y}$, P. Baillon ${ }^{j}$, D. Barney ${ }^{j}$, L. M. Barone ${ }^{\text {ac }}$, P. Barrillon ${ }^{\circ}$, A. Bartoloni $^{\text {ac }}$, S. Beauceron ${ }^{k}$, F. Beaudette ${ }^{j}$, K. W. Bell ${ }^{h}$, R. Benetta ${ }^{j}$, M. J. Bercher ${ }^{y}$, U. Berthon ${ }^{y}$, B. Betev ${ }^{a m}$,

R. Beuselinck ${ }^{\circ}$, A. Bhardwaj ${ }^{g}$, W. Bialas ${ }^{j}$, C. Biino ${ }^{\text {ag }}$, S. Bimbot ${ }^{y}$, J. Blaha ${ }^{\text {aj }}$, P. Bloch ${ }^{j}$, S. Blyth ${ }^{f}$, P. Bordalo ${ }^{m}, A$. Bornheim ${ }^{z}$, J. M. Bourotte ${ }^{y, j}$, D. Britton ${ }^{\circ}$, R. M. Brown ${ }^{h}$,

R. Bruneliere ${ }^{j}$, P. Busson ${ }^{y}$, T. Camporesi $^{j}$, N. Cartiglia ${ }^{\text {ag }}$, F. Cavallari ${ }^{\text {ac }}$, M. Cerutti ${ }^{y}$, D. Chamont ${ }^{y}$, P. Chang af ${ }^{\text {f }}$, Y. H. Chang ${ }^{f}$, C. Charlot $^{y}$, S. Chatterji ${ }^{g}$, E. A. Chen ${ }^{f}$,

R. Chipaux ${ }^{\text {k }}$, B.C. Choudhary ${ }^{g}$, D. J. Cockerill ${ }^{\text {h }}$, C. Collard ${ }^{y}$, C. Combaret ${ }^{\text {aj }}$, F. Cossutti ${ }^{\text {ah }}$, J. C. Da Silva ${ }^{\mathrm{m}}$, I. Dafinei ${ }^{\mathrm{ac}}$, , G. Daskalakis $^{\mathrm{j}}$, G. Davatz ${ }^{\text {am }}$, D. Decotigny ${ }^{\mathrm{y}}$, A. De Min ${ }^{\mathrm{p}}$, K. Deiters ${ }^{\text {ak }}, M$. Dejardin ${ }^{k}, D$. Del Re ${ }^{a c}$, R. Della Negra ${ }^{\text {aj }}, G$. Della Ricca ${ }^{a h}$, P. Depasse ${ }^{\text {aj }}$, J. Descamp ${ }^{k}$, G. Dewhirst ${ }^{\circ}$, S. Dhawan ${ }^{\text {w }}$, M. Diemoz ${ }^{\text {ac }}$, G. Dissertori ${ }^{\text {am }}$, M. Dittmar ${ }^{\text {am }}$, L. Djambazov am, L. Dobrzynski ${ }^{y}$, S. Drndarevic ${ }^{c}$, M. Dupanloup ${ }^{\text {aj }}$, M. Dzelalija ${ }^{\text {ad }}$, J. Ehlers $^{\text {am }}$, H. El Mamouni ${ }^{\text {aj }}$, A. Elliott-Peisert ${ }^{j}$, I. Evangelou ', B. Fabbro ${ }^{k}$, J. L. Faure ${ }^{k}$,

J. Fay ${ }^{\text {aj }}$, F. Ferri ${ }^{p}$, P. S. Flower ${ }^{h}$, G. Franzoni ${ }^{a}$, W. Funk ${ }^{j}$, A. Gaillac ${ }^{y}$, C. Gargiulo ${ }^{\text {ac }}$, S. Gascon Shotkin ${ }^{\text {aj }}$, Y. Geerebaert ${ }^{y}$, F. X. Gentit ${ }^{k}, A$. Ghezzi ${ }^{p}$, J. Gilly ${ }^{y}$,

A. S. Giolo-Nicollerat ${ }^{\text {am }}$, A. Givernaud ${ }^{k}$, S. Gninenko ${ }^{s}, A$. Go $^{f}$, N. Godinovic ${ }^{\text {ae }}$, N. Golubev ${ }^{s}$, I. Golutvin i, R. Gomez-Reino ${ }^{j}$, P. Govoni ${ }^{p}$, J. Grahl $^{q}$, P. Gras ${ }^{k}$, J. Greenhalgh $^{\text {h, }}$,

J. P. Guillaud ${ }^{a}$, M. Haguenauer ${ }^{y}, G$. Hamel De Montechenault ${ }^{k}$, M. Hansen ${ }^{j}$, H. F. Heath ${ }^{e}$, J. A. Hill ${ }^{\text {h, P. R. Hobson }}{ }^{\text {ai }}$, D. Holmes ${ }^{\text {e }}$, A. G. Holzner ${ }^{\text {am }}$, G. W. Hou ${ }^{\text {af }}$, B. llle ${ }^{\text {aj }}$,

Q. Ingram ${ }^{a k}$, A. Jain ${ }^{m}$, P. Jarry ${ }^{k}$, C. Jauffret ${ }^{y}$, M. Jha ${ }^{g}$, M. A. Karar ${ }^{y}$, S. K. Kataria ${ }^{v}$,

V. Katchanov ${ }^{\text {aa }}$, B. W. Kennedy ${ }^{\text {, }}$ K. Kloukinas ${ }^{j}$, P. Kokkas ', M. Korjik ${ }^{r}$, N. Krasnikov's, D. Krpic ${ }^{c}$, A. Kyriakis ${ }^{b}, M$. Lebeau $^{j}$, P. Lecomte ${ }^{a m}$, P. Lecoq ${ }^{j}$, M. C. Lemaire ${ }^{k}$,

M. Lethuillier ${ }^{\text {aj }}$, W. Lin ${ }^{f}$, A. L. Lintern ${ }^{h}$, A. Lister ${ }^{a m}$, V. Litvin ${ }^{z}$, E. Locci ${ }^{k}$, A. B. Lodge ${ }^{h}$,

E. Longo ${ }^{a c}$, D. Loukas ${ }^{b}$, D. Luckey ${ }^{a m}$, W. Lustermann ${ }^{a m}$, C. Lynch ${ }^{\mathrm{e}}$, C. K. Mackay ${ }^{\mathrm{e}}$,

M. Malberti ${ }^{\mathrm{p}}$, D. Maletic ${ }^{\mathrm{c}}$, I. Mandjavidze ${ }^{\mathrm{k}}$, N. Manthos ${ }^{\text {, A. Markou }}{ }^{\mathrm{b}}, \mathrm{H}$. Mathez ${ }^{\text {aj, }}$,

A. Mathieu ${ }^{y}$, V. Matveev ${ }^{\mathrm{s}}, \mathrm{G}$. Maurelli ${ }^{\text {aj }}, \mathrm{E}$. Menichetti ${ }^{\mathrm{ag}}, \mathrm{P}$. Meridiani ${ }^{\mathrm{ac}}, \mathrm{P}$. Milenovic ${ }^{\mathrm{c}}$,

G. Milleret ${ }^{y}$, P. Mine ${ }^{y}$, M. Mur ${ }^{k}, Y$. Musienko ${ }^{d, s}, A$. Nardulli $^{a m}$, J. Nash $^{\mathrm{j}, \mathrm{o}}, \mathrm{H} . \mathrm{Neal}^{\mathrm{w}}$,

P. Nedelec ${ }^{a}$, P. Negri ${ }^{p}$, F. Nessi-Tedaldi ${ }^{\text {am }}$, H. B. Newman ${ }^{z}$, A. Nikitenko ${ }^{\circ}$,

M. M. Obertino ag, ${ }^{\text {, }}$, R. A. Ofierzynski ${ }^{a m}$, G. C. Organtini ${ }^{a c}$, P. Paganini ${ }^{y}$, M. Paganoni $^{p}$,

I. Papadopoulos ', R. Paramatti ${ }^{\text {j, ac }}$, N. Pastrone ${ }^{\text {ag }}$, F. Pauss ${ }^{\text {am }}$, I. Puljak ${ }^{\text {ae }}$, A. Pullia ${ }^{p}$,

J. Puzovic ${ }^{c}$, S. Ragazzi ${ }^{p}$, S. Ramos ${ }^{m}, S$. Rahatlou ${ }^{\text {ac }}$, J. Rander ${ }^{k}$, K. Ranjan ${ }^{g}$, O. Ravat ${ }^{\text {aj }}$,

M. Raymond ${ }^{\circ}$, P. A. Razis ${ }^{x}$, N. Redaelli ${ }^{p}$, D. Renker ${ }^{\text {ak }}$, S. Reucroft ${ }^{d}, J_{\text {.M. Reymond }}{ }^{k}$,

M. Reynaud ${ }^{\text {aj }}$, S. Reynaud ${ }^{j}$, T. Romanteau $^{y}$, F. Rondeaux ${ }^{k}$, A. Rosowsky ${ }^{k}$, C. Rovelli ${ }^{p}$, P. Rumerio ${ }^{j}$, R. Rusack ${ }^{q}$, S. V. Rusakov ${ }^{4}$, M. J. Ryan ${ }^{\circ}$, H. Rykaczewski ${ }^{a m}$,

T. Sakhelashvili ${ }^{a k},{ }^{*}$, R. Salerno ${ }^{p}$, M. Santos ${ }^{m, n}{ }^{n}$, C. Seez ${ }^{\circ}$, I. Semeniouk ${ }^{y}$, O. Sharif ${ }^{\text {ai }}$, P. Sharp ${ }^{j}$, C. Shepherd-Themistocleous ${ }^{h},{ }^{\prime}$ S. Shevchenko ${ }^{z}$, R. K. Shivpuri ${ }^{g}$, 
G. Sidiropoulos ', D. Sillou ${ }^{\text {a }}$, A. Singovski ${ }^{\text {a }}$, Y Sirois ${ }^{y}$, A.M. Sirunyan ${ }^{\text {al }}$, B. Smith ${ }^{\text {h }}$, V. J. Smith ${ }^{e}$, M. Sproston ${ }^{h}$, H. Suter ${ }^{\text {am }}$, J. Swain ${ }^{d}$, T. Tabarelli De Fatis $^{p}$, M. Takahashi ${ }^{\circ}$, R. J. Tapper ${ }^{e}$, A. Tcheremoukhine ${ }^{i}$, I. Teixeira ${ }^{m, n}, J^{2}$ P. Teixeira ${ }^{m, n}, O$. Teller ${ }^{j}$, C. Timlin $^{\circ}$, F. A. Triantis ', S. Troshin ${ }^{a a}$, N. Tyurin ${ }^{a a}$, K. Ueno af, A. Uzunian ${ }^{\text {aa }}$, J. Varela ${ }^{\mathrm{m}, \mathrm{j}}$, N. Vaz Cardoso ${ }^{m}$, P. Verrecchia ${ }^{k}$, P. Vichoudis ${ }^{1, j}$, S. Vigano ${ }^{p}, G$. Viertel ${ }^{a m}, T$. Virdee ${ }^{0, j}$, E. Vlassov ${ }^{\mathrm{j}, \mathrm{t}}, \mathrm{M}$. Wang ${ }^{\text {af }}$, A. Weinstein ${ }^{\mathrm{z}}, \mathrm{J}$. H. Williams ${ }^{\mathrm{h}}$, I. Yaselli ai, A. Zabi ${ }^{\circ}$, N. Zamiatin ${ }^{\prime}$, S. Zelepoukine ${ }^{\text {aa }}$, M. Zeller ${ }^{\text {w }}$, L. Y. Zhang ${ }^{z}$, J. Zhang ${ }^{9}, Y^{\prime}$. Zhang ${ }^{\circ}$, K. Zhu ${ }^{z}$ and R. Y. Zhu ${ }^{{ }^{\prime}}$

a Laboratoire d'Annecy-le-Vieux de Physique des Particules, 74941 Annecy-le-Vieux, France

b Institute of Nuclear Physics "Demokritos", 15310 Attiki, Greece

c "Vinca" Institute of Nuclear Sciences and Faculty of Physics of University of Belgrade, 11000

Belgrade, Serbia

d Northeastern University, Boston MA 02115-5096, USA

e Bristol University, Bristol BS8 1TL, United Kingdom

f National Central University, Chung-Li, Taiwan, ROC

g Delhi University, Delhi 110 007, India

h CCLRC, Rutherford Appleton Laboratory, Didcot OX11 0QX, United Kingdom

i Joint Institute for Nuclear Research, 141980 Dubna (Moscow Region), Russia

j European Organization for Nuclear Research, CERN, 1211 Geneva 23, Switzerland

k CEA/Saclay, 91191 Gif-sur-Yvette Cedex, France

I University of loannina, 45110 loannina, Greece

m Laboratório de Instrumentação e Física Experimental de Partículas, 1000-149 Lisboa, Portugal

n Instituto de Engenharia de Sistemas e Computadores, 1000-029 Lisboa, Portugal

o Imperial College, London SW7 2BZ, United Kingdom

p Università degli Studi Milano-Bicocca and INFN-Sezione di Milano, 20126 Milano, Italy

q University of Minnesota, Minneapolis MN 55455, USA

r Research Institute for Nuclear Problems, Byelorussian State University, 220050 Minsk, Byelorussia

s Institute for Nuclear Research, Russian Academy of Sciences, 117312 Moscow, Russia

t Institute for Theoretical and Experimental Physics, RU-117259, Moscow

u Lebedev Physical Institute, Russian Academy of Sciences, 117924 Moscow, Russia

v Bhabha Atomic Research Centre, Mumbai 400 085, India

w Yale University, New Haven CT 06520-8121, USA

x Cyprus University, 1678 Nicosia, Cyprus

y Laboratoire Leprince-Ringuet, Ecole Polytechnique, 91128 Palaiseau Cedex, France

z California Institute of Technology, Charles C. Lauritsen Laboratory, Pasadena CA91125, USA

aa State Research Center, 142284 Protvino (Moscow Region), Russia

ab ENEA - CR Casaccia, 00060 S. Maria di Galeria, Roma, Italy

ac Università "La Sapienza", Dipartimento di Fisica and INFN-Sezione di Roma, 00185 Roma, Italy

ad Split University, PMF, 21000 Split, Croatia

ae Technical University of Split, FESB, 21000 Split, Croatia

af National Taiwan University, 106 Taipei, Taiwan ROC

ag Università di Torino, Dipartimento di Fisica and INFN-Sezione di Torino, 10125 Torino, Italy

ah Università di Trieste, Dipartimento di Fisica and INFN-Sezione di Trieste, 34127 Trieste, Italy

ai Brunel University, Uxbridge, Middlesex UB8 3PH, United Kingdom

aj Institut de Physique Nucléaire, IN2P3-CNRS and Université C. Bernard Lyon I, 69622

Villeurbanne, France

ak Paul Scherrer Institut, 5232 Villigen, Switzerland

al Yerevan Physics Institute, 375036 Yerevan 36, Armenia

am Eidgenössische Technische Hochschule, ETH, 8093 Zürich, Switzerland

* On leave from High Energy Physics Institute, Tblisi, Georgia 


\begin{abstract}
The energy resolution of the barrel part of the CMS Electromagnetic Calorimeter has been studied using electrons of 20 to $250 \mathrm{GeV}$ in a test beam. The incident electron's energy was reconstructed by summing the energy measured in arrays of $3 \times 3$ or $5 \times 5$ channels. There was no significant amount of correlated noise observed within these arrays. For electrons incident at the centre of the studied $3 \times 3$ arrays of crystals, the mean stochastic term was measured to be $2.8 \%$ and the mean constant term to be $0.3 \%$. The amount of the incident electrons' energy which is contained within the array depends on its position of incidence. The variation of the containment with position is corrected for using the distribution of the measured energy within the array. For uniform illumination of a crystal with $120 \mathrm{GeV}$ electrons a resolution of $0.5 \%$ was achieved. The energy resolution meets the design goal for the detector.
\end{abstract}




\section{Introduction}

The Compact Muon Solenoid (CMS) experiment [1] is a general purpose detector which will operate at the $14 \mathrm{TeV}$ proton-proton collider, LHC, at CERN. A main goal of the experiment is the discovery of the Higgs boson. If the Higgs mass is near the lower limit of $114 \mathrm{GeV}$ currently established [2], a favourable discovery channel is its decay into two photons. To observe this decay, the measurement of the photons' energies with high resolution and uniform response will be decisive. CMS is equipped with a hermetic homogeneous electromagnetic calorimeter (ECAL) [3] made of lead tungstate $\left(\mathrm{PbWO}_{4}\right)$ crystals. Lead tungstate has a fast response and is resistant to radiation; it has a high density $\left(8.3 \mathrm{~g} . \mathrm{cm}^{-3}\right)$, a short radiation length $\left(X_{0}=0.89 \mathrm{~cm}\right)$ and a small Moliere radius $\left(R_{M}=2.0 \mathrm{~cm}\right)$, which allows a highly granular, compact detector to be built.

The ECAL consists of a cylindrical barrel containing 61,200 crystals, closed at each end with end-caps, each containing 7324 crystals. The barrel is made up of 36 supermodules, each with 1700 crystals arranged in a quasi-projective geometry. The principal axes of the crystals are at an angle of $3^{\circ}$ to the vectors from the nominal proton-proton interaction vertex, in both the azimuthal and polar angle projections [4]. The individual crystals are truncated pyramids with a lateral size close to $R_{M}$ and a length of $25.8 X_{0}$.

In this paper, the energy resolution of the ECAL determined from data taken with electrons of 20 to $250 \mathrm{GeV}$, in October and November 2004, is discussed. The energy of the incident electrons is determined by summing the energy measured in an array of $3 \times 3$ or $5 \times 5$ crystals. However, these arrays do not fully contain the electron shower, and the amount of leakage out of the array depends on the electron's incident position. A correction for the variation of the amount of the energy contained in the array, based on the relative sizes of the signals within the array, has been developed and is also discussed in this paper.

Further details of the results presented here may be found in Refs $[5,6]$.

\section{Experimental set up}

A supermodule (SM10) was mounted on a movable, computer controlled table at the $\mathrm{H} 4$ beamline at CERN. The supermodule's position was adjusted such that beam electrons were incident at an angle of $3^{\circ}$ to the axis of a selected crystal in both transverse directions. The beam could be directed at each crystal in the supermodule.

A beam telescope defining an area of $20 \times 20 \mathrm{~mm}^{2}$, slightly smaller than the front faces of the crystals $\left(\approx 22 \times 22 \mathrm{~mm}^{2}\right)$ was used to trigger the supermodule readout. A four plane scintillating fibre hodoscope with $150 \mu \mathrm{m}$ spatial resolution was used to determine the transverse position of the incident particle. The transverse coordinates $X$ and $Y$ at the hodoscope correspond to the $\eta$ (pseudorapidity) and $\Phi$ (azimuthal) directions across the supermodule in the CMS coordinate system. Fig. 1 shows typical beam intensity profiles measured at the hodoscope.
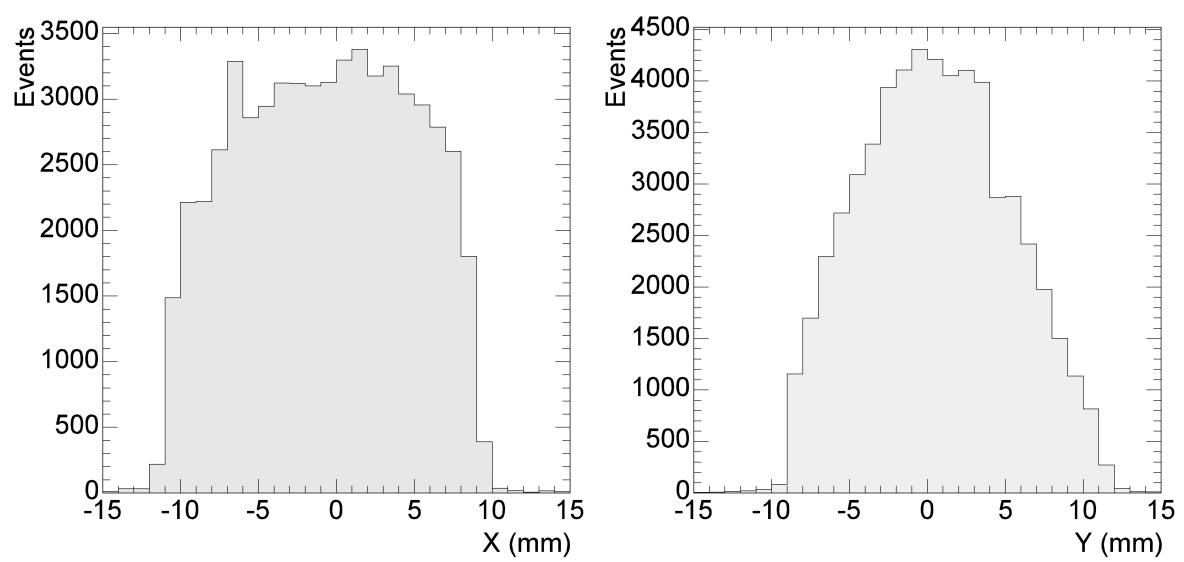

Figure 1. Beam intensity profiles in the $X$ (left) and $Y$ (right) directions 
The selected crystal was generally positioned in the beam such that the energy deposit in this crystal was maximized. Due to the $3^{\circ}$ off-pointing of the crystal axis, in this position the centre of the crystal front face does not coincide with the beam axis. Nevertheless we refer to this geometry by saying that the beam is 'centred' on the crystal. Data were also taken with the crystals moved half way between the positions of maximum response of adjacent crystals; we refer in this case to the beam being centred on the edge (or corner if moved in both directions) of the crystal, although again the edge (or corner) of the front face is not on the beam axis.

The crystals form an array of $20 \times 85$ in the $\Phi \times \eta$ directions in the supermodule, numbered such that crystals $1-20$ are at $\eta \sim 0$ in CMS and crystals $1681-1700$ are at maximum $\eta(=$ 1.479).

The supermodule was fully equipped with the final front end electronics [4] as well as with the final high and low voltage supplies, cooling and temperature systems $[7,8,9]$, and laser monitoring system [10]. Each crystal is read out independently. The light produced in each crystal is detected by a pair of avalanche photodiodes [11]. The signal from the diodes is shaped and amplified in a Multi-Gain Pre-Amplifier (MGPA) with three parallel gain stages, of nominal gains 12,6 , and 1 . The signals with all gains are sampled and digitized continuously at $25 \mathrm{~ns}$ intervals by Analogue to Digital Converters (ADCs) and the largest unsaturated ADC value is read out each time. (If the read-out switches to a lower gain as the pulse grows, the return to higher gain is delayed by five samples after the threshold is re-crossed.)

In the analysis of the test beam data, the signal amplitude is reconstructed from up to five consecutive digitized samples, using a digital filtering method which minimizes the contribution of the electronics noise [12]. For signals read out at gain 12, pedestal-subtracting weights are used, which take samples before the pulse to perform an event-by-event pedestal subtraction. For an electron with energy above about $160 \mathrm{GeV}$, the readout of the crystal on which it is incident may switch to gain 6 . To achieve good energy resolution in these cases the ratio of the two nominal gains, 6 and 12, must be precisely determined. This was done by finding the gain ratio giving the best energy resolution for data taken with $180 \mathrm{GeV}$ electrons [6]. For gain 6, the pedestal is determined from independent samples.

In order to equalize the response of each channel, an intercalibration procedure was carried out [4], using data taken with beam in the same period.

\section{Noise measurements}

The electronics noise was measured by applying the amplitude reconstruction procedure to data taken with a random trigger, when there was no incident electron signal present (pedestal events).

The distribution of reconstructed amplitudes for a single channel is shown in Fig. 2a. The width of this distribution is obtained from a Gaussian fit and is a measure of the noise. The distribution of such noise measurements for a large number of channels is shown in Fig. $2 b$. The mean single channel noise is $41.5 \mathrm{MeV}$ equivalent. This level of noise is consistent with the MGPA design goal [13]. Figures $2 \mathrm{c}$ and $2 \mathrm{~d}$ show the distributions of the measured noise in arrays of $3 \times 3$ and $5 \times 5$ crystals, which have average values of 127 and $213 \mathrm{MeV}$ equivalent, respectively, about 3 times and 5 times the single channel noise. This shows that there is little channel-to-channel correlated noise. 

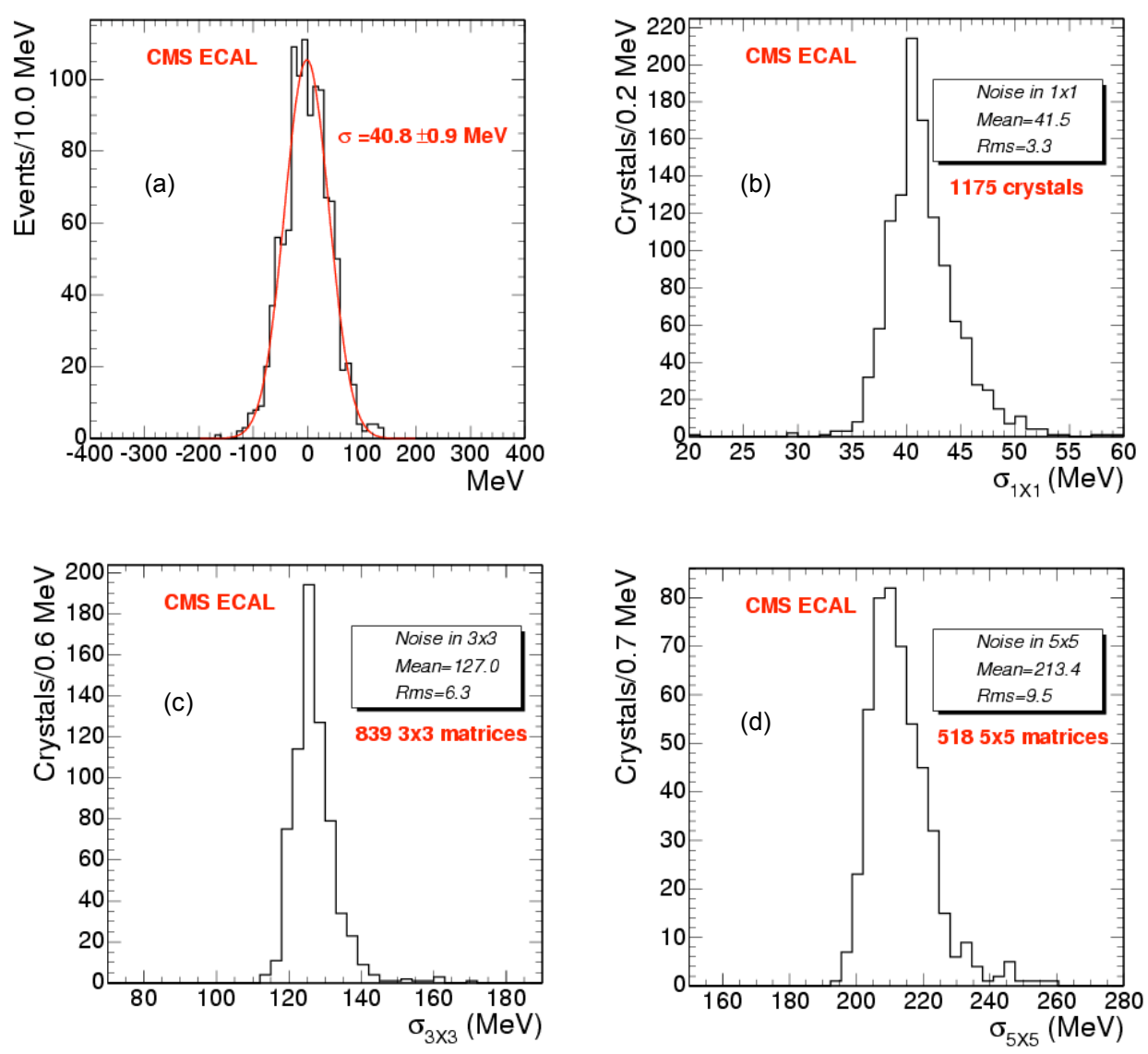

Figure 2. Measurement of the electronics noise in single crystal channels and in arrays of crystals, in MeV equivalent. a) distribution of the amplitude reconstructed for a single channel; b) distribution of noise measurements in 1175 single channels; c) distribution of the noise measured in 839 arrays of $3 \times 3$ crystals; d) distribution of the noise measured in 518 arrays of $5 \times 5$ crystals.

\section{Energy resolution for central incidence}

\subsection{Resolution at 120 and $250 \mathrm{GeV}$}

Central incidence is defined as incidence within a region $4 \times 4 \mathrm{~mm}^{2}$ around the point of maximum shower containment [5]. For electrons incident within this area the average amount of the shower energy contained in the array is almost constant. Hence using data restricted to central incidence can provide a measure of the energy resolution of the calorimeter without contributions from variations in containment losses or intercalibration errors. However, only $7 \%$ of the events taken with the $20 \times 20 \mathrm{~mm}^{2}$ trigger are within the central incidence boundaries. In this section, results are given for runs with 30,000 events taken in two crystals located at the same azimuth, but different pseudorapidity in CMS.

The spectra of energy reconstructed in arrays of $3 \times 3$ and $5 \times 5$ crystals centred on crystals 704 and 1104 are shown in Figs. 3 and 4, for 120 and $250 \mathrm{GeV}$ electrons. For these plots the mean signal amplitude was normalised to the beam energy.

A tail towards low energy can be observed in all these distributions. Nothing abnormal could be found in the signals for the events in these tails, but it is possible that they are due to electrons showering upstream in the beam line. 

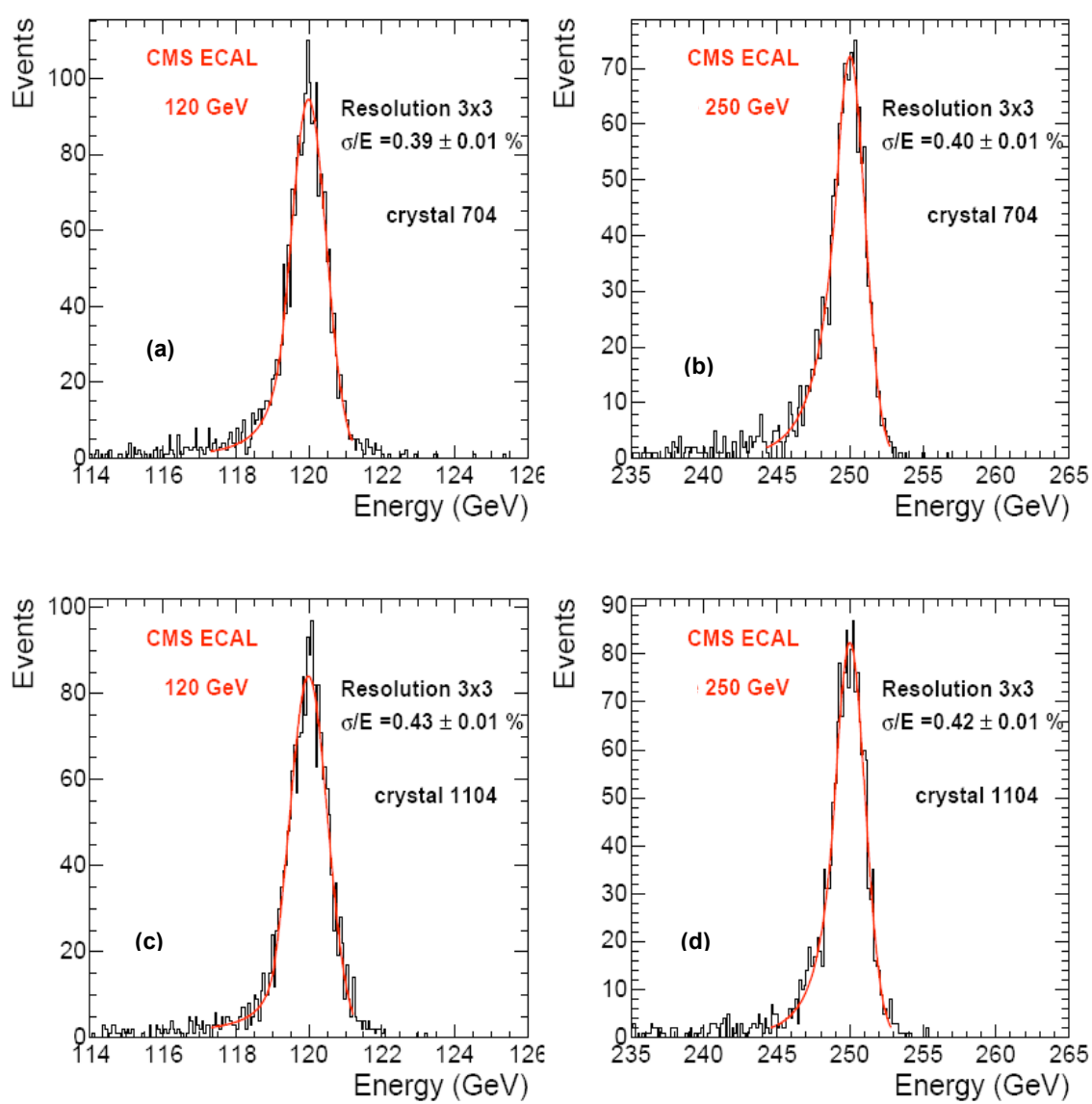

Figure 3. Distribution of energy reconstructed for central incidence in arrays of $3 \times 3$ crystals centred on crystals 704 and 1104. a) $120 \mathrm{GeV}$ electrons on crystal 704; b) $250 \mathrm{GeV}$ electrons on crystal 704 ; c) $120 \mathrm{GeV}$ electrons on crystal 1104; d) $250 \mathrm{GeV}$ electrons on crystal 1104.

The energy resolution was determined using a fit with a Gaussian combined with a polynomial tail. The resolutions obtained from the data shown in Figs. 3 and 4 are given in Table 1. All these results have uncertainties of $0.01 \%$, but have not been corrected for contributions from the energy spread of the beam.

\begin{tabular}{|l|c|c|c|c|}
\hline & \multicolumn{2}{|c|}{ 120 GeV } & \multicolumn{2}{c|}{ 250 GeV } \\
\hline & $\mathbf{3 \times 3}$ & $\mathbf{5 x 5}$ & $\mathbf{3 \times 3}$ & $\mathbf{5 \times 5}$ \\
\hline Crystal 704 & $0.39 \%$ & $0.42 \%$ & $0.40 \%$ & $0.43 \%$ \\
\hline Crystal 1104 & $0.43 \%$ & $0.43 \%$ & $0.42 \%$ & $0.41 \%$ \\
\hline
\end{tabular}

Table 1. The energy resolution measured with crystals 704 and 1104 at 120 and $250 \mathrm{GeV}$ in arrays of $3 \times 3$ and $5 \times 5$ crystals. The uncertainty on these results is $0.01 \%$. The contribution from the energy spread of the beam has not been subtracted. 

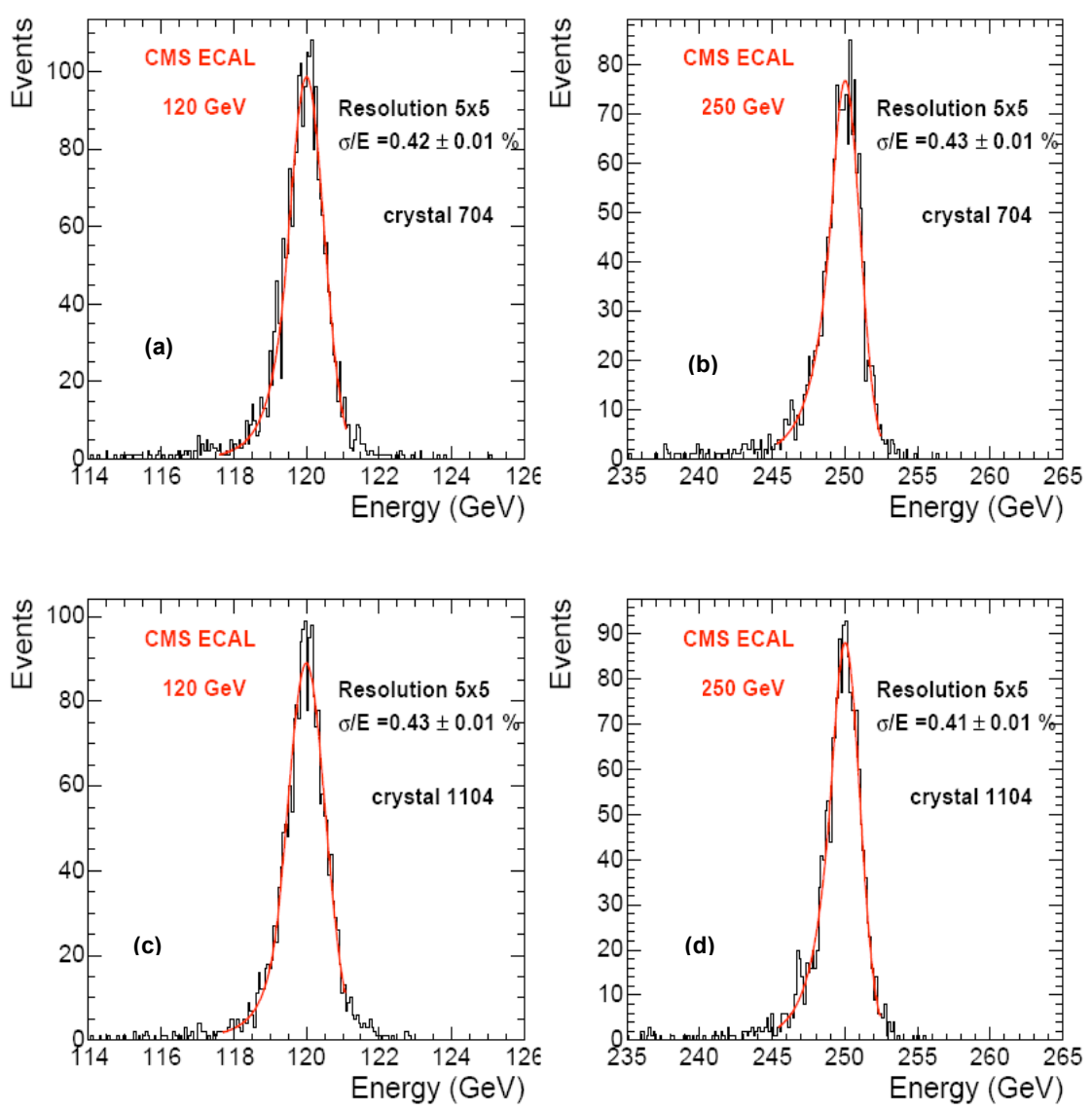

Figure 4. Distribution of energy reconstructed for central incidence in arrays of $5 \times 5$ crystals centred on crystals 704 and 1104. a) $120 \mathrm{GeV}$ electrons on crystal 704; b) $250 \mathrm{GeV}$ electrons on crystal 704; c) $120 \mathrm{GeV}$ electrons on crystal 1104; d) $250 \mathrm{GeV}$ electrons on crystal 1104.

\subsection{Resolution as a function of energy}

At all energies there is a spread in electron energy of $\pm 0.09 \%$ rms due to the beamline optics. In addition, fluctuations in synchrotron radiation make significant additional contributions to the energy spread [14] at the higher energies. These are $0.05 \%, 0.11 \%$ and $0.23 \%$ at 150 , 180 and $250 \mathrm{GeV}$, respectively, with estimated uncertainties of half these values. The results given in this section have both these contributions to the observed energy resolutions quadratically subtracted.

The energy resolution was determined for central incidence for seven electron energies between 20 and $250 \mathrm{GeV}$. The results were then fitted as a function of energy according to:

$$
\left(\frac{\sigma}{E}\right)^{2}=\left(\frac{S}{\sqrt{E}}\right)^{2}+\left(\frac{N}{E}\right)^{2}+C^{2}
$$

where $S$ is the stochastic term, $N$ the noise and $C$ the constant term, and $E$ is the energy in $\mathrm{GeV}$. The fit is performed with the noise term fixed for each crystal at the value measured in the pedestal runs.

The energy resolutions using the $3 \times 3$ arrays centred on crystal 704 and on 1104 are shown in Fig. 5, together with the fitted resolution function curves. The error bars include both statistical and systematic errors, with the statistical uncertainties becoming large at the lower energies. 

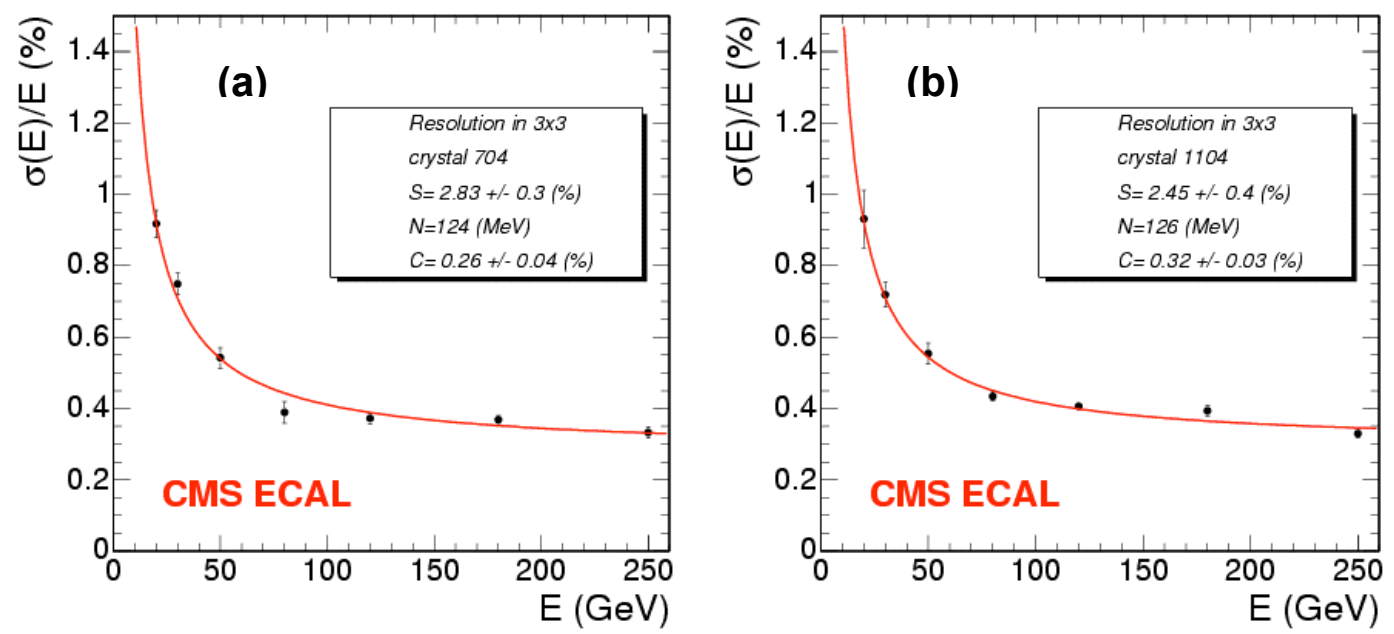

Figure 5. Resolution as a function of the energy for the $3 \times 3$ array of crystals centred a) on crystal 704 and b) on crystal 1104, with beam hodoscope cuts of $4 \times 4 \mathrm{~mm}^{2}$.

Data were also taken with the beam centred on each of the nine crystals forming the $3 \times 3$ arrays around each of the two reference crystals (704 and 1104). The mean values of the energy resolution measured for all 18 crystals are listed in Table 2, for each incident energy.

\begin{tabular}{|l|c|c|c|c|c|c|c|}
\hline Energy (GeV) & 20 & 30 & 50 & 80 & 120 & 180 & 250 \\
\hline Resolution (\%) & 0.94 & 0.74 & 0.56 & 0.45 & 0.40 & 0.38 & 0.34 \\
\hline Rms Spread (\%) & \pm 0.058 & \pm 0.044 & \pm 0.025 & \pm 0.031 & \pm 0.021 & \pm 0.026 & \pm 0.027 \\
\hline
\end{tabular}

Table 2. Mean values of the energy resolution for arrays of $3 \times 3$ crystals centred on 18 different crystals, for each incident beam energy, and the rms spread over the 18 crystals. The contributions from the energy spread of the beam have been subtracted.

The results for all 18 crystals were fitted using Eq. 1 . The values of stochastic and constant terms thus obtained are shown in Fig. 6 . The mean value of the stochastic term is $2.8 \%$ and that of the constant term is $0.30 \%$.

The values of the stochastic and constant terms are as expected from the statistical fluctuations in the number of photo-electrons produced in the APDs, the effects of longitudinal non-uniformities of the crystal response and the statistical fluctuations of the containment losses [3]. 

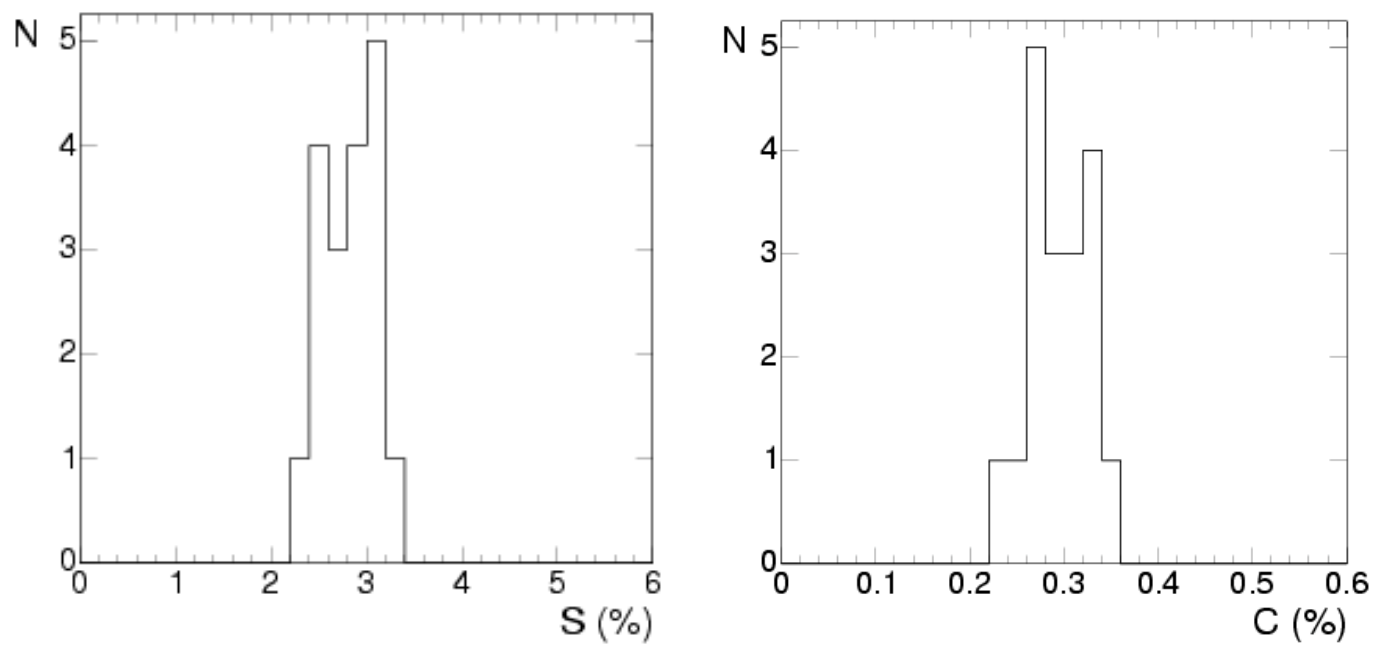

Figure 6. Stochastic (left) and constant (right) terms obtained for 18 crystals, with the energy reconstructed in $3 \times 3$ arrays.

\section{Correction for shower leakage}

The $3 \times 3$ and $5 \times 5$ arrays of crystals used to determine an incident electron's energy do not fully contain the electron shower. The amount leaking out of the array depends on the electron's incident position, due to the finite size of the array and because of the small gaps between the crystals. To achieve the desired energy resolution and uniformity of response when the incident electrons are distributed over the full crystal front face a correction must be applied on an event-by-event basis [5].
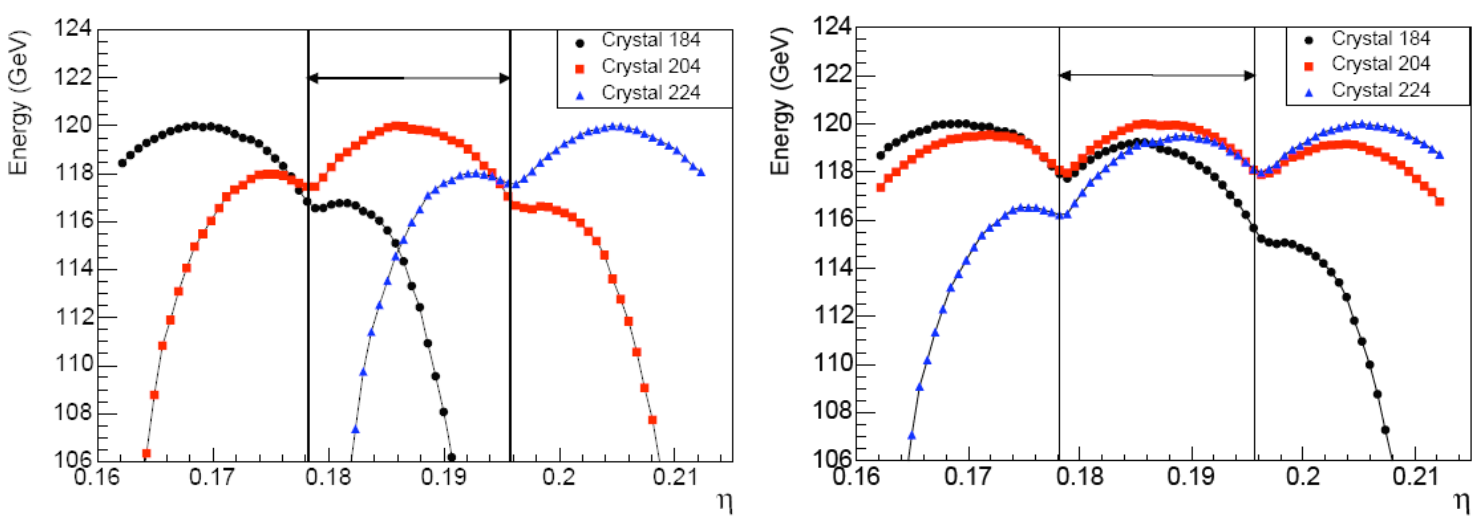

Figure 7. Left: mean energy measured in $3 \times 3$ arrays centred on crystals 184 (dots), 204 (squares), 224 (triangles) as a function of incident position, $\eta$. Right: same for $5 \times 5$ arrays. The arrows indicate the region where crystal 204 has the largest energy deposit.

The variation of the measured energy with the incident position is illustrated in Fig. 7, which shows the energy measured in the $3 \times 3$ and $5 \times 5$ arrays centred on each of three adjacent crystals as a function of incident position (in the $\eta$ direction, measured with the beam hodoscope). The maximum response has been normalized to the beam energy (120 GeV) in each case. The region of incident position where the central crystal has the largest energy deposit (indicated by the arrows) does not coincide with the position of that crystal's front face because of the $3^{\circ}$ off-pointing, which is also responsible for the asymmetries observed. For each curve the envelope is determined largely by the variation of lateral leakage out of the array, while the dips near the vertical lines show the effect of the gaps between the crystals. 
In the region indicated by the arrows (where the energy measured in the array around crystal 204 would be used) the maximum variation in the measured energy is about $2.5 \%$ for the $3 \times 3$ array and $1.6 \%$ for the $5 \times 5$ array. Similar distributions are obtained when plotting the measured energy as a function of incident position in the $\Phi$ direction.

\subsection{The correction method}

The method used to correct the non-uniform response illustrated in Fig 7 uses the distribution of energy measured in the array itself to determine the correction [5]. Corrections are determined according to the distributions in the $\eta$ and $\Phi$ directions independently. No position measurement external to ECAL is required, but instead a variable $\ln (E 2 / E 1)$ is used, which is closely related to the electron's position of incidence. Knowledge of the energy of the incident particle is also not required.

The determination of the variable $\ln (E 2 / E 1)$ is illustrated in Fig. 8 for a $3 \times 3$ array. By definition, the array is formed around the crystal with the largest energy deposit. To determine the quantities $E 1$ and $E 2$ related to the energy distribution in the $\eta$ direction, the energies measured in the single crystals on the high and low $\eta$ sides of the central crystal (W1 and W2 respectively) are compared. Then the energies measured in each of the three columns of three crystals are summed, and the sum in the central column is added to the sum in the column containing the smaller of W1 and W2. This forms the two quantities E1 and E2:

- If $\mathrm{W} 1<\mathrm{W} 2$ (Fig. 8a) $\mathrm{E} 1$ is the sum of the energy in the central and high $\eta$ columns, and E2 is the sum of the energy in the column at low $\eta$. In this case E2 <E1.

- If $\mathrm{W} 1>\mathrm{W} 2$ (Fig. 8b) E1 is the sum of the energy in the column at high $\eta$ only, and $\mathrm{E} 2$ is the sum of the energy in the central and low $\eta$ columns. In this case E2 > E1.

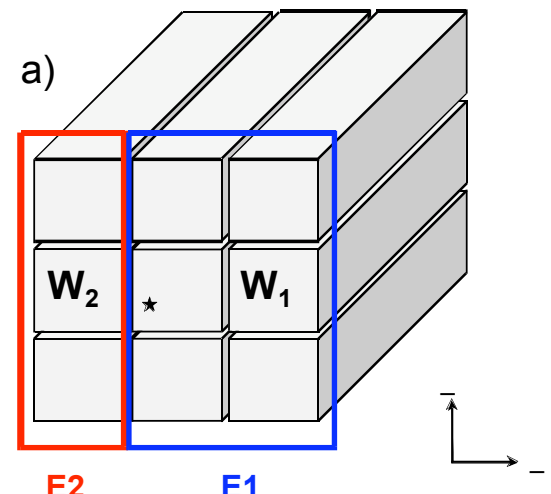

E2

E1

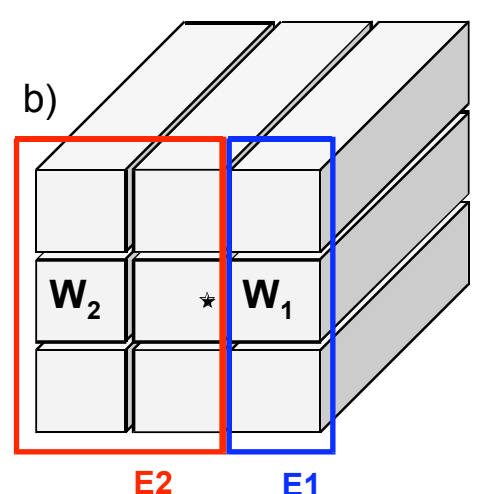

Figure 8. Definition of $E 1$ and $E 2$ when a) $W 1<W 2$ and b) $W 1>W 2$ (see text). The star represents the electron's incident position.

The sum of the energy measured in the $3 \times 3$ array is then evaluated as a function of the variable $\ln (E 2 / E 1)$. The logarithmic function is chosen because the energy density of electromagnetic showers falls off exponentially outside the shower core, making the distribution of events over $\ln (E 2 / E 1)$ more uniform than that over, for example, the simple ratio E2/E1.

Figure 9 shows the correspondence between $\ln (E 2 / E 1)$ and the spatial coordinate $X$ at the front of the crystal, as measured with the beam hodoscope. Large values of $|\ln (E 2 / E 1)|$ occur for electrons near the centre of the crystal so that most of the energy is deposited in the 
central column of the matrix, belonging either to E2 (in which case E2>>E1) or E1 (in which case $E 1>>E 2)$. For electrons incident near either edge of the crystal the energy is shared nearly equally between $E 1$ and $E 2$ and $|\ln (E 2 / E 1)|$ approaches 0.

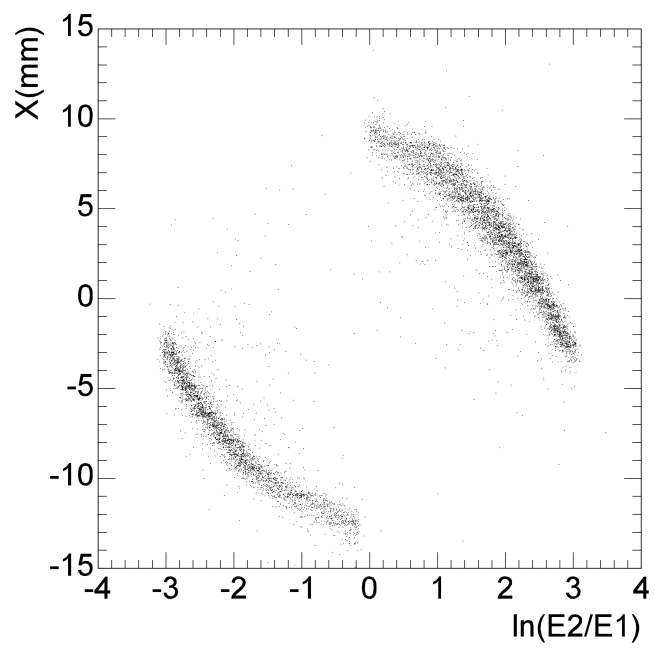

Figure 9. The parameter $\ln (\mathrm{E} 2 / \mathrm{E} 1)$ versus coordinate $X$ for $120 \mathrm{GeV}$ electrons incident on crystal 204.

$\mathrm{E} 1$ and $\mathrm{E} 2$ are determined in the same way in $\eta$ and $\Phi$. For a $5 \times 5$ array the method is similar, but the clusters used to define E1 and E2 contain 10 or 15 crystals.

To determine the correction, the mean measured energy is determined as a function of $\ln (E 2 / E 1)$, and polynomials are fitted to the resulting distribuitions. Finally these polynomials are used to correct the data on an even-by-event basis.

\subsection{Application of the method}

The mean energies of $120 \mathrm{GeV}$ electrons measured in the $3 \times 3$ and $5 \times 5$ arrays are plotted as a function of $\ln (E 2 / E 1)$ in Figs. 10 and 11, for both $\eta$ and $\Phi$ directions. The mean energy is normalized to 1 at $\ln (E 2 / E 1)=-3$. The data were obtained with the electron beam incident in turn on the centre of crystal 204 and on the middle of each of its four edges (as defined in section 2). Only events where crystal 204 contained the largest energy deposit were used. The positive and negative $\ln (\mathrm{E} 2 / \mathrm{E} 1)$ distributions are not symmetric because of the $3^{\circ}$ offpointing geometry of the crystals. The curves are the polynomials fitted to the each of the positive and negative regions of the $\eta$ and $\Phi$ distributions independently.

The polynomial functions resulting from the fits are used to correct the energy measured in the array on an event-by-event basis. For each event the product of the corrections in $\eta$ and in $\Phi$ is applied. The maximum correction is about $0.5 \%$ larger in $\eta$ than in $\Phi$ for both the $3 \times 3$ and the $5 \times 5$ arrays.

The energy reconstructed in the $3 \times 3$ array after the corrections have been applied is plotted as a function of the $X$ and $Y$ positions measured by the beam hodoscope in Fig. 12, with crystal 204 centred on the beam axis. The distributions obtained when the edge of the crystal is on the beam axis show a similar uniformity, as do all the distributions for the $5 \times 5$ array. 

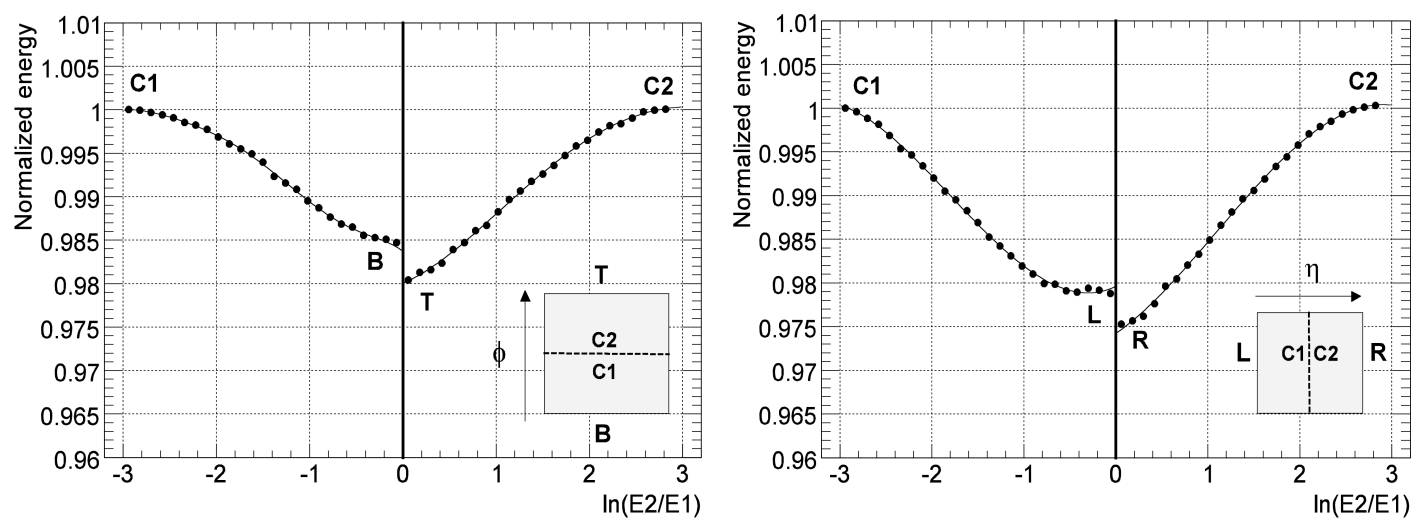

Figure 10. Normalized mean energy measured in the $3 \times 3$ array around crystal 204 versus $\ln (E 2 / E 1)$ in the $\Phi$ (left) and $\eta$ (right) directions. The curves are 3rd order polynomial functions fitted to the measured distributions, independently for positive and negative values of In(E2/E1). The square panels represent the central crystal with various regions indicated: $T$ (top), B (bottom), L (left), R (right) and C1 and C2 (just off centre on each side). The labels on the distributions indicate in which region the electrons were incident.
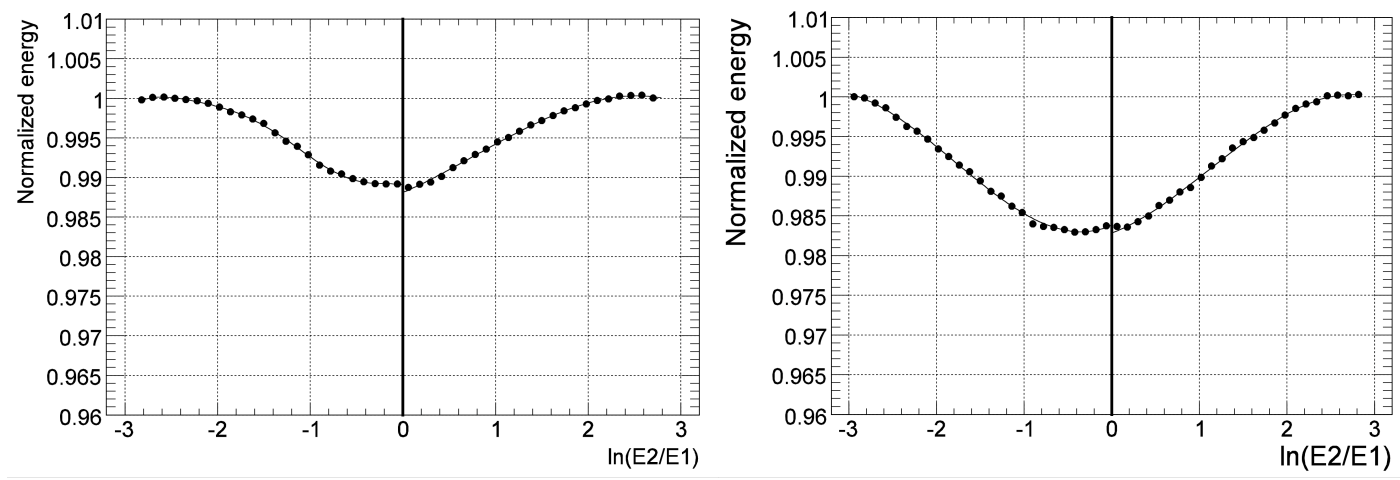

Figure 11. The same as Figure 10, but for the 5x5 array around crystal 204.
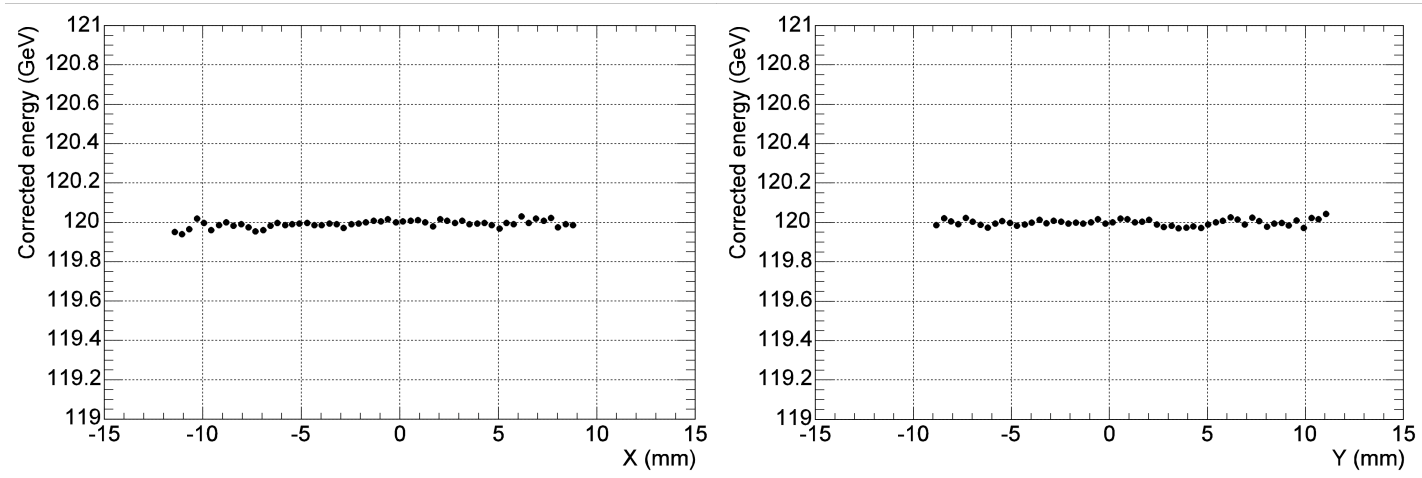

Figure 12. Corrected mean energy measured in the $3 \times 3$ array around crystal 204 versus $X$ (left) and $Y$ (right) positions as measured with the beam hodoscope. 


\subsection{Simulations}

Simulations were made using H4SIM [15] to define the geometry and GEANT4 to describe the electromagnetic showers. The results for $120 \mathrm{GeV}$ electrons are shown in Figs. 13 and 14 for the $3 \times 3$ and $5 \times 5$ arrays. The observed asymmetries between the positive and negative regions of $\operatorname{In}(E 2 / E 1)$ are well reproduced, as well as the difference in the asymmetries for the two arrays. However, the $0.5 \%$ differences in the maximum correction between the $\eta$ and $\Phi$ directions seen in the data are not reproduced. This is not yet understood.
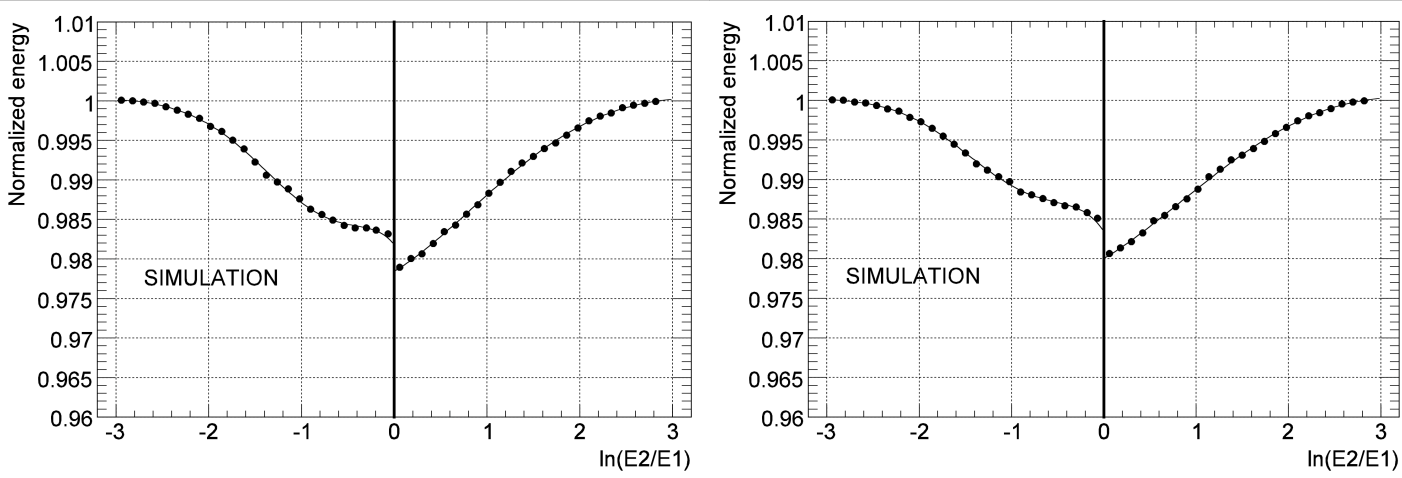

Figure 13. Simulated normalized mean energy measured in the $3 \times 3$ array around crystal 204 versus $\ln (\mathrm{E} 2 / \mathrm{E} 1)$ in the $\Phi$ (left) and $\eta$ (right) directions.
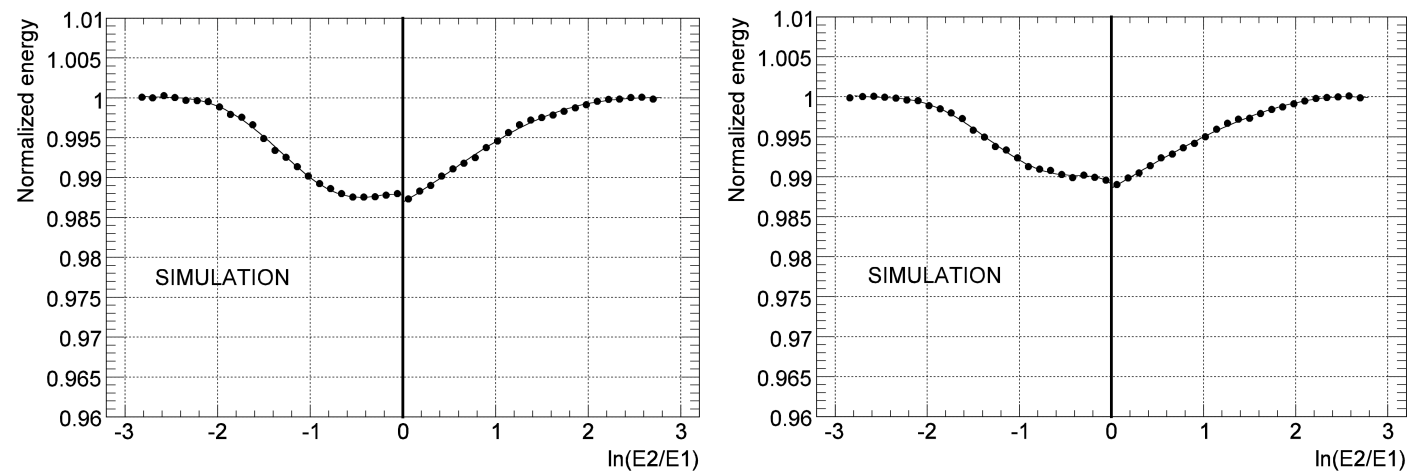

Figure 14. Simulated normalized mean energy measured in the $5 \times 5$ array around crystal 204 versus $\ln (E 2 / E 1)$ in the $\Phi$ (left) and $\eta$ (right) directions.

\subsection{Dependence on position in the calorimeter}

The data taken for crystals 204, 704 and 1104 were used to investigate the dependence of the leakage correction on the position in $\eta$ in the calorimeter. The results are shown in Fig.15 for the $3 \times 3$ arrays. In the $\eta$ view, the spread between the three sets of distributions is smaller than $\sim 0.2 \%$, while in $\Phi$ differences up to $\sim 1 \%$ are seen. Broadly similar behaviour is seen for $5 \times 5$ arrays. This dependence on position in the calorimeter is also seen in simulations, although the effects are somewhat smaller. However, these effects are neglected here and the correction functions determined for crystal 204 at $120 \mathrm{GeV}$ are used for all crystals and all incident energies. 

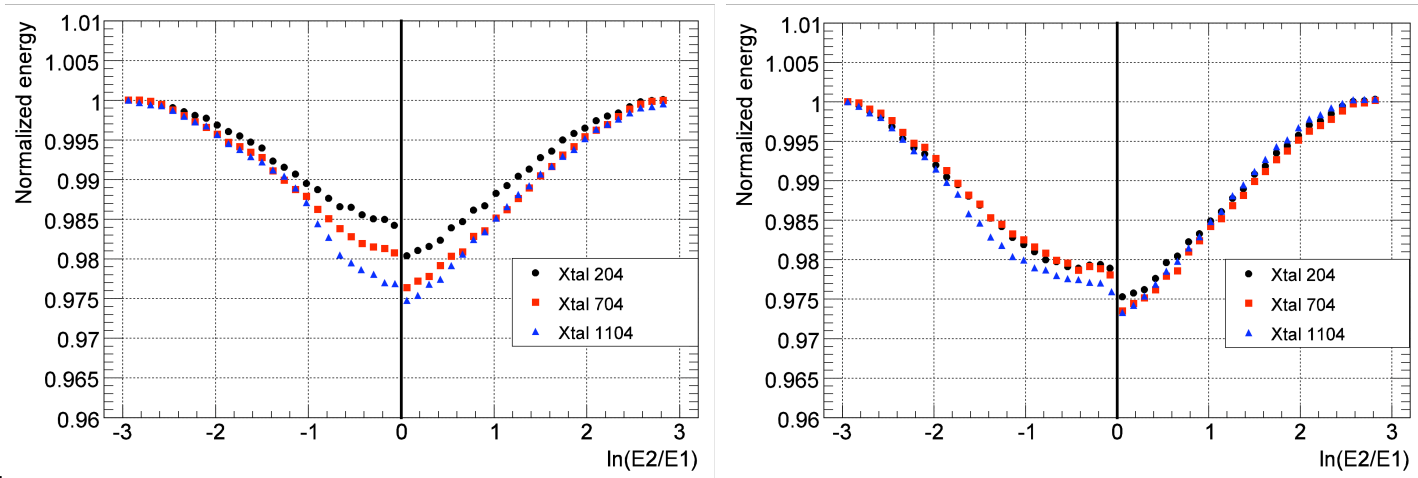

Figure 15. Normalized mean energy measured in the $3 \times 3$ arrays around crystals 204,704 and 1104 , versus $\ln (E 2 / E 1)$ in the $\Phi$ (left) and $\eta$ (right) directions.

\subsection{Energy Resolution}

Figure 16 shows the effect of the corrections on the energy resolution when $120 \mathrm{GeV}$ incident electrons are centred on crystal 204 , using the full $20 \times 20 \mathrm{~mm}^{2}$ trigger. The data were normalised such that the mean signal after the correction equals the beam energy. The corrected distribution is fitted as the sum of a Gaussian and a polynomial to describe the low energy tail. The energy resolution, for the sum in the $3 \times 3$ array, defined as the ratio of the rms of the Gaussian fit to the energy, is $0.43 \%$. In the case of the $5 \times 5$ array, it is not significantly different.
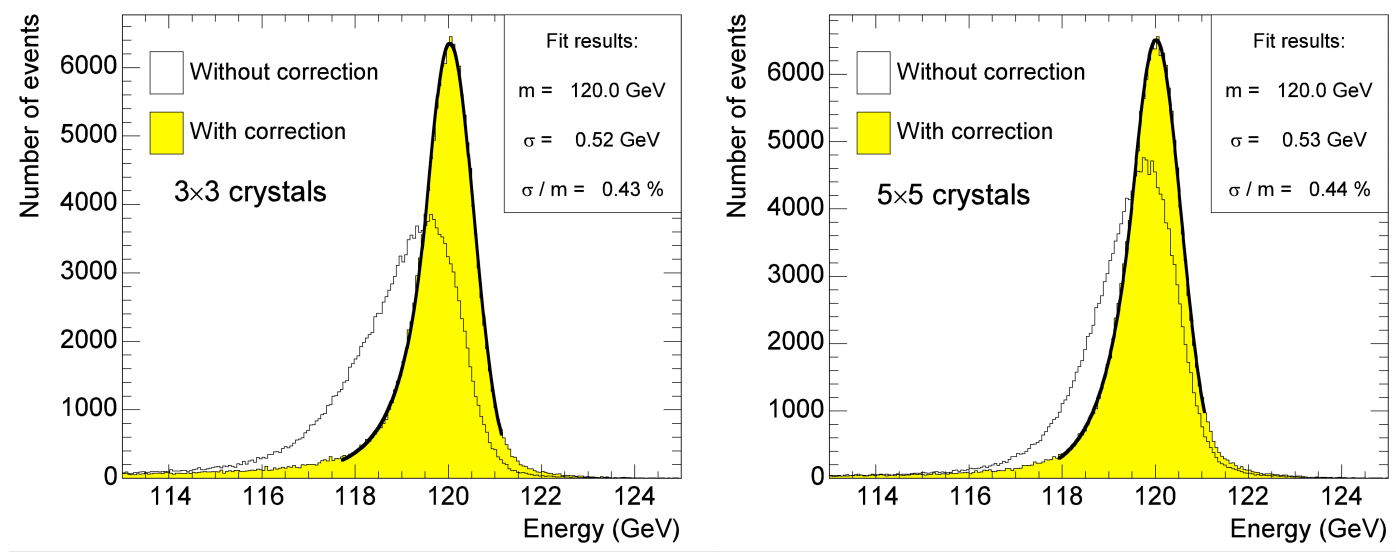

Figure 16. Distribution of measured energy before (unshaded) and after (shaded) correction for the shower leakage, for crystal 204, using (left) the $3 \times 3$ array of crystals and (right) the $5 \times 5$ array.

Figure 17 compares the energy resolution for central incidence with that using the full $20 \times 20$ $\mathrm{mm}^{2}$ trigger, with the shower leakage correction applied, as a function of incident energy, measured in the $3 \times 3$ array around crystal 1104 . Crystal 1104 was taken for this plot to be different to the crystal from which the correction functions were evaluated. The energy resolution obtained using the full trigger is about $0.1 \%$ worse than that for central incidence. 


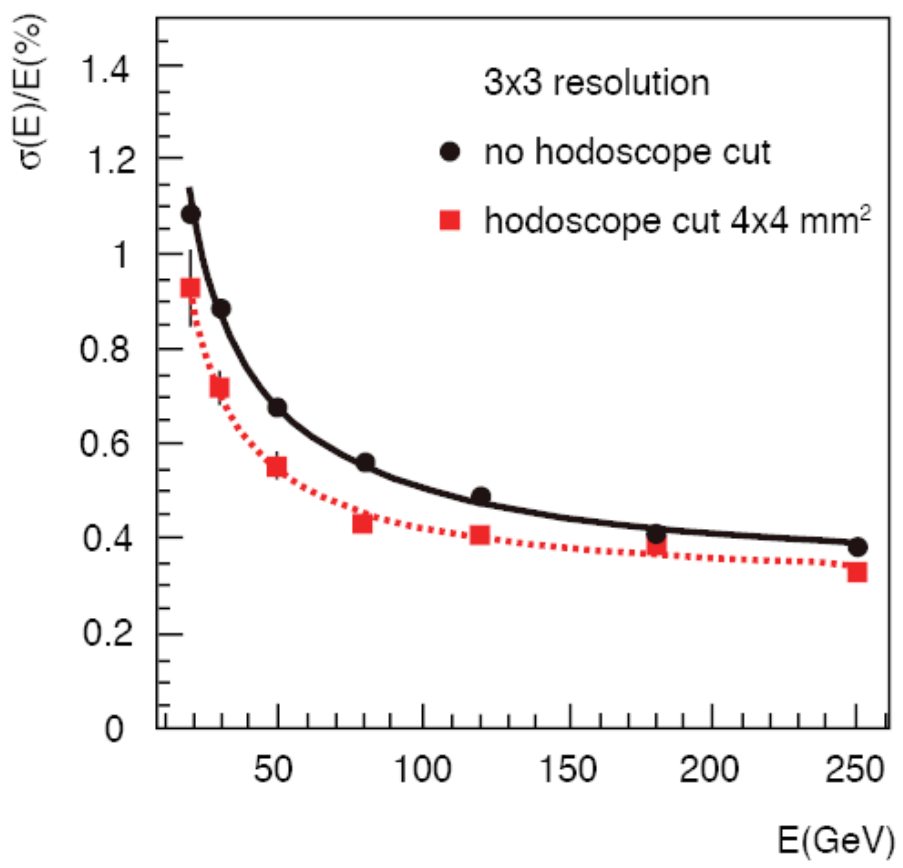

Figure 17. Energy resolution measured in the $3 \times 3$ array around crystal 1104 with (squares) and without (dots) the $4 \times 4 \mathrm{~mm}^{2}$ hodoscope cuts, as a function of incident energy, $E$.
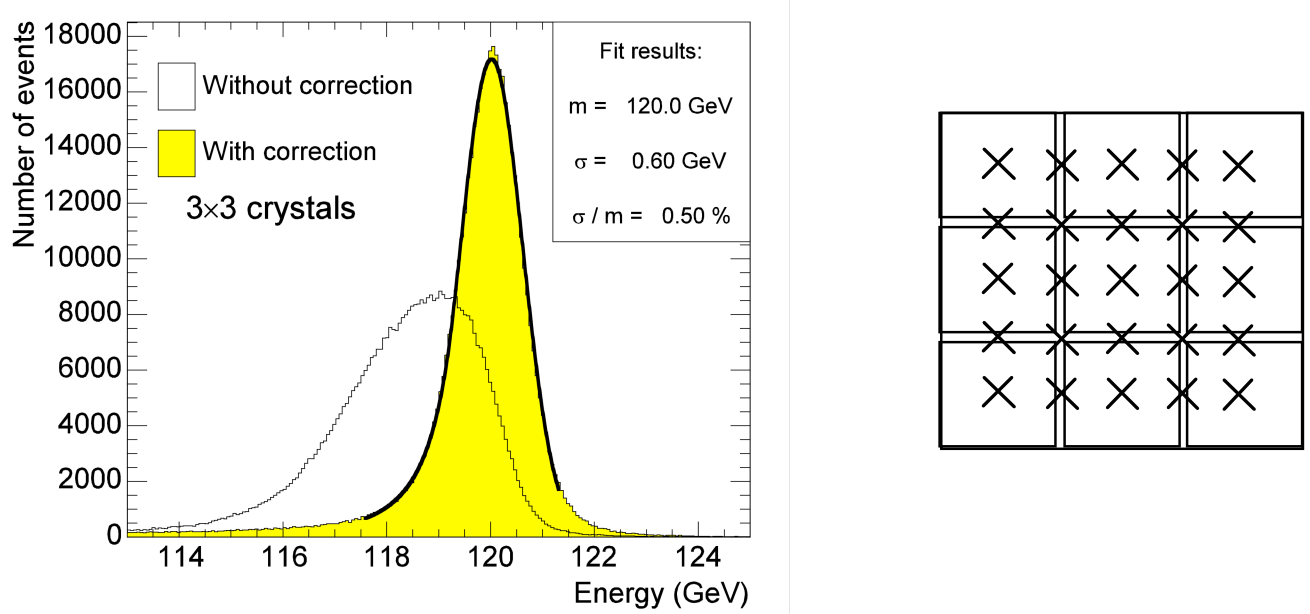

Figure 18. Left: Distribution of energy before (unshaded) and after (shaded) correction for the shower leakage when the incident beam is centred in turn at many locations in the $3 \times 3$ array of crystals around crystal 204 . The beam was centred at, and symmetrically between, the positions of maximum response of the crystals. Right: Schematic indication of these beam positions marked with crosses.

Finally, Fig. 18 shows the effect of the corrections for data in which the beam was in turn centred on the centre of all crystals in the $3 \times 3$ array around crystal 204, on all four corners of the central crystal, and on the edges between the nine crystals. (As explained in section 2, the term centre refers to the position where the shower containment in the crystal is maximum, while edge and corner refer to positions mid way between the positions of maximum containment of two crystals.) This combination, with roughly equal amounts of beam in each position, approximates uniform coverage of the array. As for Fig. 16, the data have been normalised such that the mean signal after the correction equals the beam energy. 
The measured resolution is $0.50 \%$. This value can be compared to $0.43 \%$ (Fig. 16) for electrons incident over the full $20 \times 20 \mathrm{~mm}^{2}$ trigger area centred on the crystal.

\section{Conclusions}

The average measured noise of the ECAL read-out electronics is $41.5 \mathrm{MeV}$ equivalent, meeting the design goal of the electronics, and there is no significant correlated noise in arrays of $3 \times 3$ or $5 \times 5$ crystals used to reconstruct the energy of incident electrons.

The energy resolution was measured for incident electrons of seven energies from 20 to 250 $\mathrm{GeV}$. For electrons incident within a $4 \times 4 \mathrm{~mm}^{2}$ area about the point of maximum response, the measured resolutions are consistent with expectations, with mean values of the stochastic and constant terms of $2.8 \%$ and $0.3 \%$, respectively.

A method to correct for shower leakage out of the array used to reconstruct the electron's energy has been developed, which uses only the distribution of energy measured within the array. Applying this correction for $120 \mathrm{GeV}$ electrons incident on the central crystal of an array of 9 crystals, uniformly covered by the incident electrons, results in a energy resolution of $0.50 \%$.

In CMS there will be an additional contribution to the constant term for the overall resolution from the precision of the intercalibration of all channels. However, with enough events the intercalibration errors are expected to be small [4] and the results in this paper show that the CMS ECAL should perform consistently with the design goals of the experiment.

\section{Acknowledgements}

We would like to thank and acknowledge the large community of technicians and engineers, from CERN and collaborating Institutes, who worked on the preparation of the supermodule and its setting up and operation in the test beam, as well as those setting up the equipment in the beam area.

\section{References}

[1] CMS Collaboration, "The Compact Muon Solenoid - Technical Proposal", CERN/LHCC 94-38 (1994).

[2] ALEPH, DELPHI, L3 and OPAL Collaborations, "Search for the standard model Higgs Boson at LEP”, Phys. Lett. B565 (2003) 61.

[3] CMS Collaboration, "The Electromagnetic Calorimeter Project", Technical Design Report, CERN/LHCC 97-23 (1997).

[4] CMS Collaboration, “CMS Physics Technical Design Report Vol. 1", CERN/LHCC 2006-001 (2006).

[5] J. Descamps and P. Jarry, "Periodic position dependence of the energy measured in the CMS electromagnetic calorimeter", CMS Note 2006/045 (2006).

[6] CMS Electromagnetic Calorimeter Group, P. Adzic et al., "Energy resolution performance of the CMS Electromagnetic Calorimeter", CMS Note 2006/140 (2006). 
[7] CMS Electromagnetic Calorimeter Group, P. Adzic et al., " Results of the first performance tests of the CMS electromagnetic calorimeter", Eur. Phys. J. C44, s2 (2006) 1-10.

[8] P. Milenovic, J. Puzovic, D. Jovanovic, D. Maletic, G. Dissertori and P. Adzic, "Performance of the CMS ECAL safety system for Super Modules SM0 and SM1", Nucl. Instrum. Meth. A554 (2005) 427-436.

[9] P. Adzic et al., Proceedings of the $10^{\text {th }}$ International Conference on Accelerator and Large Experimental Physics Control Systems, Geneva 2005, ICALEPCS, MO3.4-10 (2005); CMS Conference Report CR 2005/028 (2005).

[10] L. Zhang, D. Bailleux, A. Bornheim, K. Zhu, R.Y. Zhu, "Performance of the Monitoring Light Source for the CMS Lead Tungstate Crystal Calorimeter", IEEE Trans. Nucl.Sci. 52 (2005) 1123-1130.

[11] Z. Antunovic et al., "Radiation hard avalanche photodiodes for the CMS detector", Nucl. Instrum. Meth. A537 (2005) 379, and references therein.

[12] CMS Electromagnetic Calorimeter Group, P. Adzic et al., "Reconstruction of the signal amplitude of the CMS electromagnetic calorimeter", Eur. Phys. J. C46, s1 (2006) 23-35.

[13] M. Raymond, J. Crooks, M. French and G. Hall, "The MGPA Electromagnetic Readout Chip for CMS", in Proceedings of the $9^{\text {th }}$ Workshop on Electronics for the LHC Experiments, 2003, CERN-LHCC-2003-055 (2003).

[14] P. Grafstrom, " The momentum resolution of the $\mathrm{H} 4$ beam for secondary beams in Filter Mode optics", CERN Accelerator and Beams SL-Note-97-81/EA (1997).

[15] T. Frisson and P. Mine, "H4SIM, a Geant4 simulation program for the CMS ECAL supermodule". Presented at the Geant $410^{\text {th }}$ international Conference, Bordeaux, November 2005.

URL=http://geant4.in2p3.fr/2005/Workshop/UserSession/P.Mine.pdf 